\title{
Optical and Near-Infrared Observations of Classical Nova V1723 Aql
}

\author{
M. Nagashima ${ }^{1}$, A. Arai ${ }^{1}$, M. Isogai ${ }^{1}$, T. Arasaki ${ }^{1}$, E. Kitao ${ }^{1}$, \\ G. Taguchi ${ }^{1}$, Y. Ikeda ${ }^{1}$, H. Kawakita ${ }^{1}$, M. Yamanaka ${ }^{2}$, R. Itoh ${ }^{2}$, \\ M. Sasada ${ }^{2}$, T. Okushima ${ }^{2}$, M. Uemura ${ }^{2}$, K. S. Kawabata ${ }^{2}$, \\ H. L. Worters ${ }^{3}$, and D. P. Smits ${ }^{4}$ \\ ${ }^{1}$ Kyoto Sangyo University, Motoyama Kamigamo Kita-ku Kyoto Kyoto, Japan \\ email: g0837714@cc.kyoto-su.ac.jp \\ ${ }^{2}$ Hiroshima University, 1-3-1 Kagamiyama Higashi-Hiroshima Hiroshima, Japan \\ ${ }^{3}$ South African Astronomical Observatory, PO Box 9, Observatory 7935, South Africa \\ ${ }^{4}$ Department of Mathematical Sciences, University of South Africa, Box 392, UNISA, \\ Pretoria 0003, South Africa
}

\begin{abstract}
Optical and near-infrared observations of novae give us useful information for understanding the diversity of nova eruptions. Classical nova V1723 Aql was discovered by F. Kabashima and K. Nishiyama on 2010 September 11. We have conducted photometric and spectroscopic observations of V1723 Aql in both optical and near-infrared (NIR) wavelength regions since its discovery. The $V$-band decline time by 2 mag after the maximum, $\mathrm{t}_{2}$, was $\sim 12 \mathrm{~d}$. The apparent Fe II emission lines were also seen in the optical spectra. The $R_{\mathrm{c}^{-}}$and $I_{\mathrm{c}}$-band light curves exhibited rapid declines $\left(0.16 \mathrm{mag} \mathrm{d}^{-1}\right.$ in $\left.R_{\mathrm{c}}\right) 20$ days after the visual maximum, while the $\operatorname{NIR}\left(J, H\right.$, and $\left.K_{\mathrm{s}}\right)$ showed slow decline rates $\left(\sim 0.07 \mathrm{mag} \mathrm{d}^{-1}\right)$. This rapid reddening suggests that dust particles formed during the very early phase of the expansion in V1723 Aql.
\end{abstract}

Keywords. stars: novae, cataclysmic variables — dust, extinction

In a close binary system which consists of a white dwarf and a star (whose mass is smaller than that of the white dwarf's progenitor and fills its Roche-lobe), the gas donated to the white dwarf can ignite run-away nuclear fusion of hydrogen, causing a nova explosion. The explosion can produce significant mixing that can leave an observable imprint on the ejected material. Recently, several dust forming novae were observed soon after explosion, and the dust-formation in such early phases could be recognized. In those novae, significant dust formation was recognized $\sim 10$ days after their brightness maximum (e.g., V1280 Sco, Das et al. 2008). Their dust shells were considered optically thin. However, dust-formation in the early phase of the nova explosion is still unexplained.

Our spectra were obtained between 2010 September 11 and 2010 October 10 using the $1.3 \mathrm{~m}$ Araki telescope (+LOSA, the resolution power $R \sim 580$ ) at Koyama Astronomical Observatory (KAO), the $1.5 \mathrm{~m}$ Kanata telescope (+HOWPol, $R \sim 400$; Kawabata et al. 2008) at Higashi-Hiroshima Astronomical Observatory (HHAO), and the $1.9 \mathrm{~m}$ Radcliffe telescope (+Cassegrain grating spectrograph, $R \sim 1000$ ) at South African Astronomical Observatory (SAAO). Photometric data were obtained at Higashi-Hiroshima Astronomical Observatory using the $1.5 \mathrm{~m}$ Kanata telescope with TRISPEC (Watanabe et al. 2005) and HOWPol. We also took optical photometric data from archival data of VSNET.

Figure 1 shows our optical and near-infrared light curve. The maximum in $V$-band is on 2010 September $11(t=0)$ at $V=16.02 \pm 0.2$. The $V$-band decline time by $2 \mathrm{mag}$ after the maximum $\left(\mathrm{t}_{2}\right)$ is about 12 days. Based on the MMRD relation (Cohen 1985 


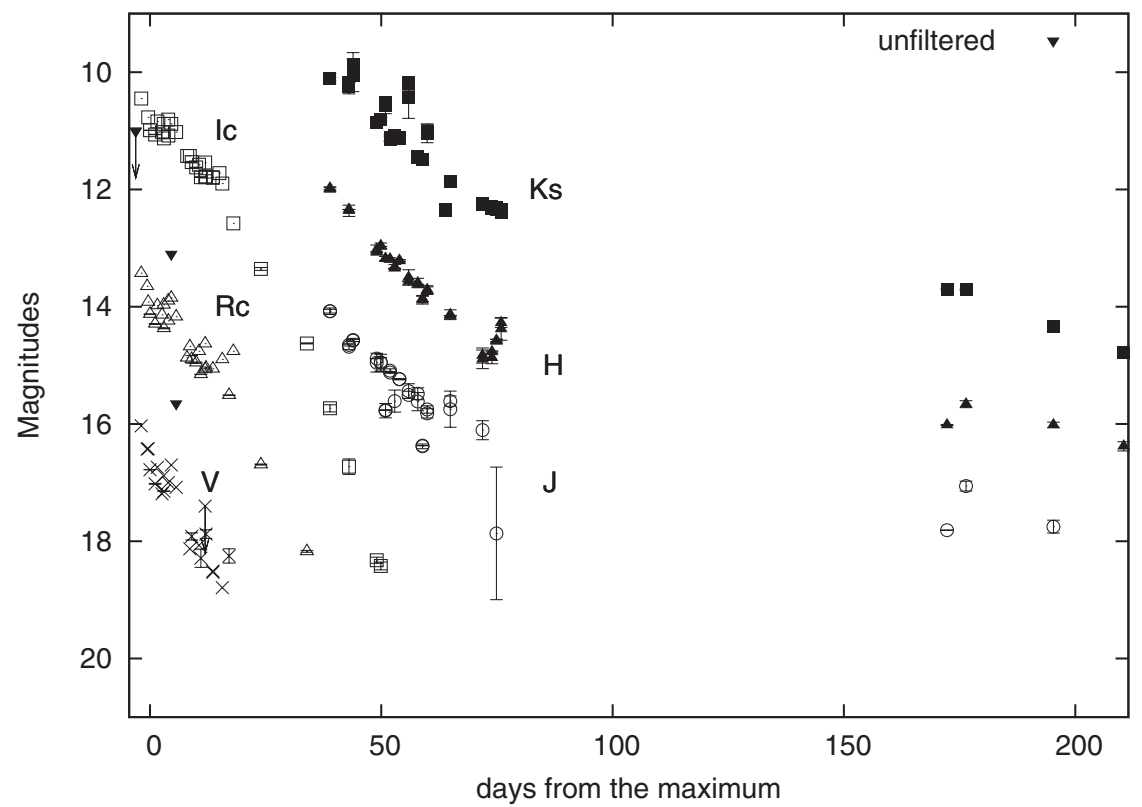

Figure 1. Optical and near-infrared light curves of V1723 Aql. The horizontal axis indicates days after maximum.

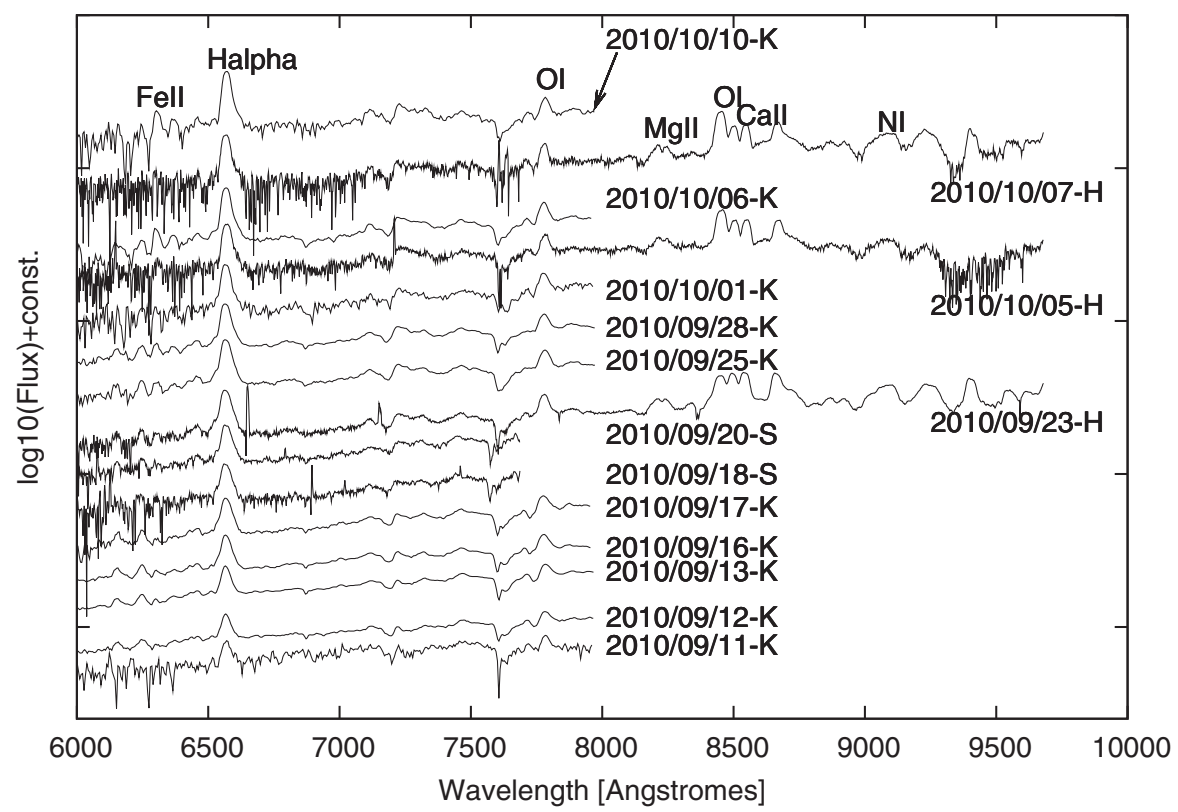

Figure 2. The evolution of nova spectra from 2010 September 11 to October 10. "K", "H", and "S" in the legends indicate KAO, HHAO, and SAAO, respectively.

and Downes \& Duerbeck 2000), we estimate $\mathrm{M}_{V} \sim-8.3$. This result suggests that the nova is relatively bright among galactic novae.

At about $t \sim 20 \mathrm{~d}$, the decline rate changed from $\Delta R_{\mathrm{c}}=0.08 \mathrm{mag} \mathrm{d}^{-1}$ to $\Delta R_{\mathrm{c}}=0.15$ mag d ${ }^{-1}$ and from $\Delta I_{\mathrm{c}}=0.06 \mathrm{mag} \mathrm{d}^{-1}$ to $\Delta I_{\mathrm{c}}=0.24 \mathrm{mag} \mathrm{d}^{-1}$. While the decline rates in the $J, H$ and $K_{\mathrm{s}}$ bands were about $0.07 \mathrm{mag} \mathrm{d}^{-1}$ even after $t \sim 20 \mathrm{~d}$. The change in colors 
(reddening) between the optical bands $\left(R_{\mathrm{C}^{-}}\right.$and $I_{\mathrm{C}^{-}}$-bands) and the near-infrared bands ( $J_{-}, H$-, and $K_{\mathrm{s}}$-bands) implies the dust formation after $t \sim 20$ days. In Figure 2 , the emission lines of Fe II and O I were recognized in these spectra, so this nova is classified into the Fe II-type nova. SAAO spectra on September 18 and 20 show an asymmetric profile of $\mathrm{H} \alpha$ emission line, which could be reproduced by two Gaussian components ($190 \mathrm{~km} \mathrm{~s}^{-1}$ and $\left.360 \mathrm{~km} \mathrm{~s}^{-1}\right)$. This indicates a nonspherically symmetric distribution of ejecta. We estimated an opacity of the dust shell on $t=39$ days. An opacity of the dust shell surrounding the pseudo-photosphere is described by $\tau \sim L_{\mathrm{IR}} / L_{\mathrm{opt}}$ (where, $L_{\mathrm{opt}}$ and $L_{\mathrm{IR}}$ are de-reddened luminosities of the maximum optical and infrared wavelengths). We estimated the opacity of the dust shell assuming that the constant luminosity phase continues. The interstellar reddening is estimated assuming that a pseudo-photosphere around the maximum light would be higher than $6,000 \mathrm{~K}$. The opacity seems to be much smaller than unity $\left(\tau \ll 10^{-2}\right)$. This implies that the dust shell is in clumpy distributions or else is optically thin.

\section{References}

Cohen J. D., 1985 ApJ, 292, 90

Das R. K., 2000 MNRAS, 391, 1874

Downes R. A. \& Duerbeck H. W., 2000 ApJ, 120, 2007

Kawabata K. S. et al., 2008 SPIE, 7014E, 151

Watanabe M. et al., 2005 PASP, 117, 870 